95. Nienhuis, G.: 1981, J. Phys. B 14, p. 3117.

96. Nienhuis, G.: 1982, J. Phys. B 15, p. 535.

97. Julienne, P.S.: 1982, Phys. Rev. A 26, p. 3299.

98. Julienne, P.S. and Mies, F.H.: 1984, Phys. Rev. A 30, p. 831.

99. Hutchinson, M. and George, T.F.: 1982, Molec. Phys., 46, p. 81.

100. Hutchinson, M. and George, T.F.: 1983, J. Phys. Chem. 87, p. 2037.

101. Mies, F.H.: 1981, in "Theoretical chemistry advances and perspectives", Vol. 6B, p. 127, Acad. Press Inc.

S. Saha1-Brechot

\title{
B. COLLISIONAL CROSS SECTIONS
}

Because of the magnitude and diversity of material on electron and heavyparticle collisions, I gave references only to published papers of obvious immediate relevance to astronomical research.

\section{Electron Collistons}

\subsection{ELECTRON IMPACT IONIZATION}

A valuable compilation of semi-empirical cross sections and rate coefficients for electron impact lonization of a wide range of lons, expressed in convenient analytical form has been prepared(1). An empirical formula for K-shell ionization cross sections was derived(2). Theoretical estimates ' 233 for the tontzation of complex ions were given $(3,4)$. Experimental values were obtained for $\mathrm{Al}^{+}(5), \mathrm{He}(6), \mathrm{Fe}^{+}(7), \mathrm{Mg}^{+}, \mathrm{Ca}^{+}$and $\mathrm{Sr}^{+}(8), \mathrm{Be}^{+}(9), \mathrm{Ne}^{3+}, \mathrm{Ar}^{3+}, \mathrm{Kr}^{3+}, \mathrm{Xe}^{3+}(10 \mathrm{a})$, $\mathrm{Mg}^{+}, \mathrm{Al}^{2+}$ and $\mathrm{Si}^{3+}(10 \mathrm{~b})$, and $\mathrm{B}^{+}, \mathrm{C}^{2+}$ and $\mathrm{O}^{4+}(11)$. Ionization rate coefficients were measured for NeVI, NeVII, OVI and Ti IX(12) and for nitrogen 1ons(13). Distorted wave calculations for the fonization of FeX and the 1ron lons from FeXV through FeXXVI were reported(14).

\section{Electron Impact Excitation}

An extensive bibliography of data on electron impact excitation (and ionization) was published(15). Many calculations of excitation cross-sections of varying degrees of sophistication were carried out for positive ions since the last IAU report: Inner shell excltation of helium-1ike fons(16), lithium-1ike fons $(17 \mathrm{a}, \mathrm{b})$ and beryllium-like ions(18) were studied. More detalled calculations were performed for $\operatorname{SiIX}(19,20)$, OIV(21), $\mathrm{NeV}(20,22), \quad \operatorname{CaXV}(23), \quad \operatorname{CaXVII}(24)$, SIII(25), SiX(26), SXII(27), $\operatorname{OVII}(28,29), \mathrm{CV}$ and $\operatorname{MgXI}(30), \operatorname{sV}(31), \mathrm{FeXXV}(32)$,

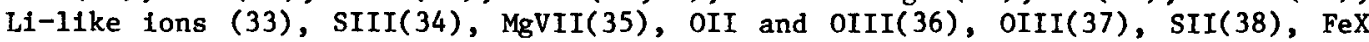
and FeXV-XXVI(14), NeIII(39), NIII(40), FeXX(41), CaXVII(42) and CII(43). Cross sections for excitation to high $n$ states of hydrogenic tons have been obtained using the Born approximation(44). Excltation of hydrogen, helium, lithium and beryllium-1ike lons has been studied(45) and of sodiulm-like Ions $\ln (46)$.

Electron impact excitation rates for neutral helium are given 1 (n) $47-50)$ and for neutral hydrogen in 51,52$)$. Fits to many excitation rate coefficients are given $\ln (53-55)$. Studies of excitation to auto-ionizing levels and satellite line emission are described in(56-67). Experimental work on electron impact excitation may be found in $(64-68)$.

A valuable compilation of electron impact excitation rate coefficients of astrophysically interesting systems has been assembled by Mendoza(69) and a useful survey of low energy collisions with complex atoms and lons has been presented by Burke and Eissner(70). 


\subsection{RECOMBINATION}

Dielectronic recombination of selected ions has received considerable experimental attention(71-73). Theoretical work has continued also(61,74-82). A tabulation of recombination coefficients by Nussbaumer and Storey(83) is of particular importance to models of astrophysical nebulae. Empirical values of the recombination coefficients of Fe X-Fe XIX have been inferred from an analysis of plasma electron densities(84).

Several measurements of the rate coefficients for the dissociative recombination of molecular ions have been reported(85-90). Theoretical studies of $\mathrm{H}_{2}{ }^{+}(91)$ and $\mathrm{H}_{3}{ }^{+}(92)$ recombination were performed with the conclusion that both are unusually slow.

\subsection{ELECTRON-MOLECULE COLLISIONS}

I 11st only references to the rotational, vibrational and electronic excitation and dissociative attachment of molecular hydrogen by electron Impact (93-100).

\section{Heavy-Particle Colliaions}

\subsection{CHARGE TRANSPER AT LOW BNERGIES}

Some further calculations on low energy charge transfer cross sections of multicharged lons in collistons with atomic hydrogen and helium(101-116) have been completed. Emptrical values for $0^{3+}$ in $\mathrm{H}$ were derived from observations of planetary nebulae(117). Estimates of charge transfer between lons of oxygen and sulphur with the neutral parents were made (118). Several measurements were reported(119-126) though only those of Church and Holzscheiter(126) refer to thermal energies.

Major reviews of theoretical charge transfer cross sections for partially and fully-stripped lons in hydrogen have appeared $(127,128)$.

\subsection{PROTON IMPACT EXCITATIONS}

A review of proton Impact cross sections was published(129) and calculations of the excitation cross-sections for fine-structure excitation were carried out $(130,131)$.

\subsection{CHEMICAL REACTIONS}

Lists of chemical reactions which play a role in interstellar circumstellar and shock region chemistry have been assembled in many papers(132-147). Extensive experimental investigations of relevant lon-molecule reactions have been reported(148-171). Theoretical analyses of the important reaction of $\mathrm{C}^{+}$with $\mathrm{H}_{2}$ have been done $(172,173)$. The possible significance of reactions producing and being driven by hot lons or atoms in cold clouds has been raised(174). The connection between endothermic and association reactions has been explored(175).

Radiative association processes are important in molecular clouds. The theory has been developed in(176-180).

A great varlety of neutral reactions that may be important in astronomical environment has been studied experimentally(181). A partial 1isting is references(181-196). Useful reviews are found in(197) and (198). Theoretical considerations are presented $\ln (199-202)$ and the dependence of the reaction rate on the rotational populations has been investigated(204). Many studies have been made of the reaction of atomic hydrogen and deuterium with $\mathrm{H}_{2}, \mathrm{HD}$ and $\mathrm{D}_{2}$, and of the influence of rotational and vibrational excitation(205-224). 


\subsection{MOLECULAR EXCITATION}

Rotational, vibrational and fine-structure excitation of astrophysically interesting molecules are discussed in(225-252). Transitions in $\mathrm{OH}$ are investigated in(253-258).

Collisions of ions with heteronuclear molecules have been studied particularly by Sakimoto(259) (see a1so 260-263).

\subsection{MOLECULAR DISSOCLATION}

The collision-induced dissociation of $\mathrm{H}_{2}$ and of $\mathrm{CO}$ has been investigated theoretically(264-267) and the rate coefficients have been shown to depend on density.

\subsection{EXCITATION BY: NEUTRAL ATOM IMPACT}

The cross section for the excitation of $O\left({ }^{3} \mathrm{P}\right)$ to $O\left({ }^{1} \mathrm{D}\right)$ by hydrogen atom impact has been calculated by Federman and Shipsey(268).

\section{References}

1. Be11, K.L., Gilbody, H.B., Hughes, J.G., Kingston, A.E. and Snith, F.J.: 1983, J. Phys. Chem. Ref. Data 12, p. 891.

2. Casnati, E., Tartari, A. and Baraldi, C.: 1982, J. Phys. B 15, p. 155.

3. Burgess, A. and Chidichimo, M.C.: 1983, MNRAS 203, p. 1269; Chidichimo, M.C.: 1983, J. Phys. B 506.

4. Shevelko, V.P., Urnov, A.M., Vainshtein, L.A., and Muller, A.: 1983, 203 p. 45 .

5. Montague, R.G., Harrison, M.F.A.: 1983, J. Phys. B 16, 3045.

6. Montague, R.G., Harrison, M.F.A. and Smith, A.C.H.: 1984, J. Phys. B 17, p. 3295 .

7. Montague, R.G., Dissens, M.J. and Harrison, M.F.A.: 1984, J. Phys. B 17 p. 2085.

8. Shrivastava, S.K. and Roy, B.M.: 1984, J. Phys. B 17, p. 4935.

9. Falk, F.A. and Dunn, G.H.: 1983, Phys. Rev. A 27, p. 754.

10a Gregory, D.C., Dittner, P.F. and Crandall, D.H.: 1983, Phys. Rev. A 27, P. 724 .

lob Crandal1, D.H., Phaneuf, R.A., Falk, R.A., Belic, D.S. and Dunn, G.H.: 1982, Phys. Rev. A 25, p. 154 .

11. Falk, R.A., Stefanis, G., Camilloni, R., Dunn, G.H., Phaneuf, R.A., Gregory, D.C. and Crandall, D.H.: 1983, Phys. Rev. A 28, p. 91.

12. Datla, U. and Roberts, J.R.: 1983, Phys. Rev. 28, p. 2201.

13. Brown, R., Deuchars, W.M., Kidd, D.E., Summers, H.P. and Wood, L.: 1983, J. Phys. B 16, p. 2053.

14. Mann, J.B.: 1983, Atom. Data Nuc1. Data Tables 29, p. 407.

15. Itikawa, Y., Takayanagi, K. and Iwai, T.: 1984, Atom. Data Nucl. Tables 31 p. 215 .

16. Sampson, D.H., Goett, S.J. and Clark, R.E.H.: 1983, Atom. Data Nucl. Data Tables 28, p. 299.

17a Goett, S.J. and Sampson, D.H.: 1983, Atom. Data Nuc1. Data Tables 29, p. 535 .

17b Goett, S.J., Sampson, D.H. and Clark, R.E.H.: 1984, Ap. J. Supp1. 54, p. 115.

18. Sampson, D.H., Goett, S.J. and Clark, R.E.H.: 1984, Atom. Data Nucl. Data Tables 30 , p. 125 .

19. Aggarwal, K.M. and Baluja, K.L.: 1983, J. Phys. B 16, p. 107.

20. Aggarwal, K.M.: 1984, Ap. J. Supp1. 55 p. 1.

21. Hayes, M.A.: 1983, J. Phys. B 16, p. 285; 1982, MNRAS 199, p. 49.

22. Aggarwal, K.M.: 1983, J. Phys. B 16, p. 2405.

23. Aggarwa1, K.M.: 1983, J. Phys. B 16, p. 2231.

24. Dufton, P.L., Kingston, A.E. and Scott, N.S.: 1983, J. Phys. B 16, p. 3053. 
25. Mendoza, C.: 1982, J. Phys. B 15, p. 867; Ho, Y.K. and Henry, R.J.W.: 1984 Ap. J. 283 , p. 816 .

26. Saha, H.P. and Trefftz, E.: 1982, J. Phys. B 15, p. 1089.

27. Saha, H.P. and Trefftz, E.: 1982, Zeits.f. Naturf. 37a, p. 744.

28. Kingston, E.E. and Tayal, S.S.: 1983, J. Phys. B 16, p. 3465.

29. Tayal, S.S. and Kingston, A.E.: 1984, J. Phys. B 17, p. 3465.

30. Tayal, S.S. and Kingston, A.E.: 1984, J. Phys. B 17, L.145.

31. Dufton, P.L. and Ktngston, A.E.: 1984, J. Phys. B 17, p. 3321.

32. Pradhan, A.K.: 1983, Phys. Rev. A 28 , p. 243.

33. Bhadra, K. and Henry, R.J.W.: 1982, Phys. Rev. A 26, p. 1848.

34. Ho, Y.K. and Henry, R.J.W.: 1984, Ap. J. 284, p. 435.

35. Aggarawa1, K.M.: 1984, Ap. J. Supp1. 56, p. 303.

36. Ho, Y.K. and Henry, R.J.W.: 1983, Ap. J. 264, p. 733.

37. Aggarwal, K.M.: 1983, Ap. J. Supp1. 52, p. 387; Aggarwa1, K.M., Baluja, K.L. and Tul1y, J.A.: 1982, MNRAS 201, p. 923.

38. Ho, Y.K. and Henry, R.J.W.: 1982, Ap. J. 267, p. 888.

39. Butler, K. and Mendoza, C.: 1983, MNRAS 208, p. 17.

40. Nussbaumer, H. and Storey, P.J.: 1982, Astron. Ap. 106, p. 271.

41. Mason, H.E. and Bhatia, A.K.: 1983, Astron. Ap. Supp1. 52, p. 181.

42. Mason, H.E. and Bhatia, A.K.: 1983, Astron. Ap. Supp1. 52, p. 115.

43. Hayes, M.A. and Nussbaumer, H.: 1982, Astron. Ap. 139, p. 233.

44. Oza, D.H.: 1984, Ap. J. Supp1. 54, p. 395; Oza, D.H., Callaway, J. and AbuSalbi, N.: 1982, Phys. Rev. A 25 p. 2812.

45. Peek, J.M. and Mann, J.B.: 1982, Phys. Rev. A 25, p. 154.

46. Henry, R.J.W. and Msezane, A.Z.: Phys. Rev. A 26, p. 2545.

47. Berrington, K.A., Burke, P.C., Fon, W.C. and Kingston, A.E.: 1982 MNRAS 200, p. 347.

48. Berringjton, K.A., Fon, W.C. and Kingston, A.E.: 1982, MNRAS 200, p. 347.

49. Freitas, L.C.G., Berrington, K.A., Burke, P.G., Hibbert, A., Kingston, A.E. and Sinfallam, A.L.: 1984, J. Phys. B 17, L303.

50. Aggarwal, K.M., Kingston, A.E. and McDowe11, M.R.C.: 1983, Ap. J. 278, p. 874 .

51. Rau, A.P.: 1984, J. Phys. B 17, L75.

52. Badnel1, N.R.: 1983, J. Phys. B 16, p. 3263.

53. Drake, S.A.: 1983, MNRAWS 205, p. 15.

54. Aggarwal, K.M.: 1983, MNRAS 202, p. 15; erratum: 1984, MNRAS 205, p. 447.

55. Stepney, S. and Gullbert, P.W.: 1983, MNRAS 204, p. 1269.

56. Clark, R.E.H., Magee, N.H., Mann, J.B. and Merts, A.L.: 1982, Ap. J. 254, p. 412 .

57. Trefftz, E.: 1983, J. Phys. B 16, p. 1247.

58. Pradhan, A.K.: 1983, Phys. Rev. A 28, p. 2128.

59. Griffin, D.C., Bottcher, C. and Pindzola, M.S.: 1982, Phys. Rev. A 25, p. 154 .

60. Foucher, P., Loulergue, M., Steenman-Clark, L. and Volonté, S.: 1982, Astron. Ap. 118 , p. 147 .

61. Faucher, P. and Volonté, S.: 1982, Astron. Ap. 135, p. 152.

62. Bely-Dubau, F., Dubau, J., Faucher, P. and Gabriel, A.H.: 1982, MNRAS 98, p. 239.

63. Bely-Dubau, F., Faucher, P., Steenman-Clark, L., Dubau, J., Loulergue, M., Gabriel, A.H., Antonucc1, E., Volonté, S. and Rapley, C.G.: 1982 MNRAS 201, p. 1158 .

64. Wang, J-S., Datla, R.U. and Griem, H.R.: 1984, Phys. Rev. A 29, p. 1558.

65. Chang, C.C., Greve, P., Kolk, K-H and Denze, H-J.: 1984, Phys. Scripta 29, p. 132 .

66. Wang, J-S and Griem, H.R.: 1983, Phys. Rev. A 27, p. 2249.

67. Kolbe, G., Huang, Y.W. and Burgess, D.D.: 1982, J. Phys. B. 15, p. 4283.

68. Johnston, A.R. and Burrow, P.D.: 1983, J. Phys. B 15, p. 613.

69. Mendoza, C.: 1983, IAU Symp. 103 (ed. D.R. Flower) p. 143.

70. Burke, P.G. and Eissner, W.: 1982, in "Atoms in Astrophysics" Ed. P.G. Burke, D.G. Hummer and I.C. Perciva1, Plenum. 
71. Williams, J.F.: 1984, Phys. Rev. A 29, p. 2936.

72. Mitche11, J.B.A., Ng, C.T., Forand, J.L., Levac, D.P., Mitchel1, R.E., Sen, A., Miko, B. and McGowan, J.W.: 1984, Phys. Rev. Lett. 50 p. 335.

73. Dittner, P.F., Datz, S., Miller, P.D., Moak, C.D., Stelson, P.H., Bottcher, C., Press, W.D., Alton, G.D., Neskovic, N. and Fox, C.M.: 1984, Phys. Rev. Lett. 51, p. 31 .

74. McLaugh1in, D.J. and Hahn, Y.: 1983, Phys. Rev. A 28, p. 493.

75. Hahn, Y., Gattuta, K.L. and McLaugh1in, D.J.: 1982, Phys, Rev. A 26, p. 1385.

76. Nussbaumer, H. and Storey, P.J.: 1982, Astron. Ap. 126, p. 75.

77. Nussbaumer, H. and Storey, P.J.: 1984, Astron. Ap.

78. McLaugh1i1n, D.J. and Hahn, Y.: 1983, J. Phys. B 16, L739.

79. Seaton, M.J.: 1984, J. Phys. B 17, L531.

80. Hickman, A.P.: 1984, J. Phys. B 17, L101.

81. LaGattuta, K.J.: 1984, Phys. Rev. A 30, p. 3072.

82. Alber, G., Cooper, J. and Rau, A.R.P.: 1984, Phys. Rev. A 30, p. 2845.

83. Nussbaumer, H. and Storey, P.J.: 1984, Astron. Ap. Supp1. 56, p. 293.

84. Isler, R.C., Crune, F.C. and Arnurius, D.E.: 1982, Phys. Rev. A 26, p. 2105.

85. MacDonald, J.A., Biond1, M.A. and Johnsen, R.: 1984, Planet. Spa. Sc1. 32, p. 651 .

86. Mitche11, J.B.A., Forand, J.L., Ng, C.T., Levac, D.P., Mitchell, R.E., Mu1, P.M., Claeys, W., Sen, A. and McGowan, J.W.: 1983, Phys. Rev. Lett. 51, p. 885 .

87. Smith, D. and Adams, W.G.: 1984, Ap. J. Lett. 284, L13; Adams, N.G., Smith and Alge, E.: 1984, J. Chem. Phys. 81, p. 1778 .

88. Mul, P.M., McGowan, J.W. Defrance, P. and Mitchel1, J.B.A.: 1983, J. Phys. B 16 , p. 3099.

89. Mitche11, J.B.A., Ng., C.T., Forand, L., Janssen, R. and McGowan, J.W.: 1984 , J. Phys. 17, L909.

90. Alge, E., Smith, D. and Adams, N.G.: 1983, J. Phys. B 16, p. 1433.

91. Giusti-Suzor, A., Bardsley, J.W. and Derkits, C.: 1983, Phys. Rev. A 28, p. 682 .

92. Michels, H.H. and Hobbs, R.H.: 1984, Ap. J. Lett. 286, L27.

93. Kurawaki, J. and Ogawa, T.: 1983, Chem. Phys. Lett. 98, p. 12.

94. Higo, M., Kamata, S. and Ogawa, T.: 1982, Chem. Phys. 66, p. 243 and 73, p. 99.

95. Morrison, M.A., Feldt, A.N. and Saha, B.C.: 1984, Phys. Rev. A 30, p. 2811.

96. Luna, M.A.P., Gibson, T.L., Taktasuka, K. and McKoy, V.: 1984, Phys. Rev. A 30 , P. 1741 .

97. Morrison, M.A., Feldt, A.N. and Austin, D.: 1983, Phys. Rev. A 29, p. 2518.

98. Wadehra, J.M.: 1983, Phys. Rev. A 29, p. 106.

99. Mu-Tao, L., Lucchese, R.R. and McKoy, V.: 1982, Phys. Rev. A 26, p. 3240.

100. Holley, T.K., Chung, S., Lin, C.C. and Lee, F.T.P.: 1983, Phys. Rev. A 26, p. 1852 .

101. Fritsch, W. and Lin, C.D.: 1984, J. Phys. B 17, p. 3271.

102. Hanssen, J., Gayet, R., Hare1, C. and Salin, A.: 1984, J, phys. B 17, L323.

103. Hardie, D.J.W. and O1son, R.E.: 1983, J. Phys. B 16, 1983.

104. Mandal, C.R., Dalta, S. and Mukherjee, S.C.: 1984, Phys. Rev. A 30, p. 1104.

105. Janev, R.K., Belic, D.S. and Bransden, B.H.: 1983, Phys. Rev. A 28, p. 1310.

106. Heil, T.G., Butler, S.E. and Dalgarno, A.: 1983, Phys. Rev. A 27, p. 2365.

107. Felckert, C.A., Blint, R.J., Surratt, G.T. and Watson, W.D.: 1984, Ap. J. 286, p. 371 .

108. Gargaud, M., McCarroll, R. and Valiron, P.: 1982, Astron. Ap. 106, p. 197.

109. Janev, R.K.: 1983, Comm. Atom. Molec. Phys. 22, p. 277.

110. Bottcher, C. and Heil, T.G.: 1982, Chem. Phys. Lett. 86, p. 506.

114. Grizdanov, T.P. and Belic, D.S.: 1984, Phys. Scripta 30, p. 194.

115. Butler, S.E., Heil, T.G. and Dalgarno, A.: 1984, J. Chem. Phys. 80, p. 4986.

116. Kimura, M. et al.: 1984, J. Phys. Soc. Japan 53, p. 2224.

117. Shields, G.A., Dalgarno, A. and Sternberg, A.: 1983, Phys. Rev. A 28, p. 2137 . 
118. Johnson, R.E. and Strobel, D.F.: 1982, J. Geophys. Res. 87, p. 10385.

119. Yosh1da, J. and O-ohata, K.: 1983, J. Phys. Soc. Japan 52, p. 417.

120. Suzuki, H., Kaj1kawa, Y., Toshurna, N., Ryufuku, H. and Watadre, T.: 1984, Phys. Rev. A 29, p. 525 .

121. Suzuki, H., Toshimo, N., Watanbe, T. and Ryufuku, H.: 1984, Phys. Rev. A 29, p. 529 .

122. Phaneuf, R.A.: 1983, Phys. Rev. A 28, p. 1310.

123. Iwai, T., Kaneko, Y., Kimuira, M., Kobayashi, N., Ohtami, S., Okimo, K., Takagi, S., Tawara, H. and Tsurubuchi, S.: 1982, Phys. Rev. A 26, p. 105 .

124. Tsurubuch1, S. et a1.: 1982, J. Phys. B 15, L733.

125. Okimo, K. et a1.: 1983, Phys. Rev. A 28, p. 127.

126. Church, D.A. and Holzscheiter, A.M.: 1982, Phys. Rev. Lett. 49, p. 643.

127. Gallagher, J.W., Bransden, B.H. and Janev, R.K.: 1983, J. Phys. Chem. Ref. Data 12, p. 873.

128. Janev, R.K., Bransden, B.H. and Gallagher, J.W.: 1983, J. Phys. Chem. Ref. Data 12, p. 829.

129. Dalgarno, A.: 1983 in Atoms in Astrophysics, Ed. P.G. Burke, W.S. Eissner, D.G. Hummer and I.C. Percival (Plenum) p. 103.

130. Heil, T.G., Kirby, K. and Dalgarno, A.: 1982, Phys. Rev. A 27, p. 2826.

131. He11, T.G., Green, S. and Dalgarno, A.: 1982, Phys. Rev. A 26, p. 3293.

132. Mitche11, G.F.: 1984, Ap. J. 287, p. 665 .

133. Thorne, L.R., Anicich, V.G., Prasad, S.S. and Huntress, W.T.: 1984, Ap. J. 280 , p. 139.

134. Herbst, E.: 1983, Ap. J. Supp1. 53, p. 41.

135. Graede1, T.E., Langer, W.D. and Frerking, M.A.: 1982, Ap. J. Suppl. 48, p. 321 .

136. Mitchel1, G.F. and Devau, T.J.: 1983, Ap. J. 266, p. 646.

137. Prasad, S.S. and Huntress, W.T.: 1983, Ap. J. 260, p. 590.

138. Suzuki, H.: 1983, Ap. J. 272, p. 579.

139. Yung, Y.L., Allen, M. and Pinto, J.P.: 1984, Ap. J. Supp1. 55, p. 465.

140. Leung, C.M., Herbst, E. and Huebner, W.F.: 1984, Ap. J. Supp1. 56, p. 231.

141. Mitche11, G.F.: 1984, Ap. J. Supp1. 54, p. 81 .

142. Nejaid, L.A.M., Millar, T.J. and Freeman, A.: 1982, Astron. Ap. 134, p. 129.

143. Millar, T.J.: 1982, MNRAS 199, p. 309.

144. Clegg, R.E.S., van 1jzendroon, L.J. and Allamondola, L.J.: 1983, MNRAS 203, p. 129.

145. Watt, G.D.: 1983, MNRAS 205, p. 321.

146. Mitchel1, G.F.: 1983, MNRAS 205, p. 765.

147. Millar, T.J. and Freeman, A.: 1983, MNRAS 207, 405 and 425.

148. Adams, N.G. and Smith, D.: 1983, in Kinetics and Energetics, Ed. A. Fontijn and M.A.A. Clyne (Academic Press) p. 31.

149. Rowe, B.R., Dupeyrot, G., Marquette, J.B., Smith, D., Adams, N.G. and Ferguson, E.E.: 1984, J. Chem. Phys. 80, p. 241 .

150. Miller, T.M., Welterskog, R.E. and Paulson, J.F.: 1984, J. Chem. Phys. 80, p. 4922 .

151. O'Keefe, A., Parent, D., Mauclaire, G. and Bowers, M.T.: 1984, J. Chem. Phys. 80 , p. 4901 .

152. Tanaka, K., Kato, T., Guyon, P-M. and Koyano, I.: 1984, J. Chem. Phys. 80, p. 4441 .

153. Smith, D., Adams, N.G. and Alge, E.: 1983, J. Chem. Phys. 78, p. 1261.

154. Barlow, S.E., Dunn, G.H. and Schauer, M.: 1984, Phys. Rev. Lett. 52, p. 1610 .

155. Herbst, E., Smith, D. and Adams, N.G.: 1982, Astron. Sp. 138, L13.

156. Kusonoki, I. and Ottinger, Ch.: 1984, Chem. Phys. Lett, 109, p. 554.

157. Adams, N.G. and Smith, D.: 1984, Chem. Phys. Lett. 105, p. 604.

158. Tanaka, K., Kato, T., Guyon, P.M. and Koyano, J.: 1983, J. Chem. Phys. 79, P. 4302.

159. Guyer, D.R., Huwe 1, L. and Leone, S.R.: 1983, J. Chem. Phys. 79, p. 1259. 
160. Chatham, H., Hils, D., Robertson, R. and Gallagher, A.C.: 1983, J. Chem. Phys. 79, p. 1301 .

161. Kemper, P.R., Bowers, M.T., Parent, D.C., Mauclaire, G., Deva1, R. and Marx, R.: 1983, J. Chem. Phys. 79, p. 1301, 79, p. 160.

162. Haudon, M., Capp, N.W. Wareing, D.P., Jones, J.D.C., Birkinshaw, K., and Twiddy, N.: 1982, J. Chem. Phys. Lett. 88, p. 63.

163. Smith, D., Adams, N.G. and Alge, E.: 1982, Ap. J. 263, p. 123.

164. Smith D., Adams, N.G. and Herbst, E.: 1983, Ap. J. 272, p. 365.

165. Herbst, E., Adams, N.G. and Smith, D.: 1982, Ap. J. 269, p. 329.

166. Federer, W., Villinger, H., Howorka, F., Lindinger, W., Tos1, P., Bassi, D. and Ferguson, E.: 1984, Phys. Rev. Lett. 52, p. 2084.

167. Illies, A.J., Jarrold, M.F. and Bowers, M.T.: 1982, J. Chem. Phys. 77, P. 5847 .

168. Bass, L.M. and Bowers, M.T.: 1982, Organ. Mass. Spectr. 17, p. 229.

169. Homa, K., Kato, T., Tanaka, K. and Koyano, J.: 1984, J. Chem. Phys. 81, p. 5666 .

170. Turner, T. and Lee, Y.T.: 1984, J. Chem. Phys. 81, p. 5638.

171. Turner, T., Dubuit, O. and Lee, Y.T.: 1984, J. Chem. Phys. 81, p. 3475.

172. Chesnavich, W.J., Akin, V.e. and Webb, D.A.: 1984, Ap. J. 287, p. 676.

173. Erwin, K.M. and Armentrout, P.B.: 1984, J. Chem. Phys. 80, p. 2978.

174. Adams, N.G., Smith, D. and Millar, T.J.: 1984, MNRAS 211, p. 857.

175. Ferguson, E.E., Smith, D. and Adams, N.G.: 1984, J. Chem. Phys. 81, p. 742.

176. Bates, D.R.: 1983, Ap. J. Lett. 267 , L121.

177. Bates, D.R.: 1983, Ap. J. 270 , p. 511 .

178. Graff, M.M., Moseley, J.T. and Roueff, E.: 1983, Ap. J. 269, p. 796.

179. Herbst, E.: 1982, Chem. Phys. 65, p. 185.

180. Freed, K.F., Oka, T. and Suzuki, H.: 1982, Ap. J. 263, p. 718.

181. Black, G., Sharpless, R.L. and Slanger, T.G.: 1982, Chem. Phys. Lett. 90 p. 55 .

182. Black, G., Sharpless, R.L. and Slanger, T.G.: 1982, Chem. Phys. Lett, 93 p. 598 .

183. Clyne, M.A.A. and Ono, Y.: 1983, Chem. Phys. Lett. 94, p. 597.

184. Ravinshankara, A.R. and Thompson, R.L.: 1983, Chem. Phys. Lett. 99, p. 327.

185. Whyte, A.R. and Phillips, L.F.: 1983, Chem. Phys. Lett. 98, p. 590.

186. Sridharan, U.C. and Kaufman, F.: 1983, Chem. Phys. Lett. 102, p. 45.

187. Black, G., Jusinski, L.E. and Slanger, T.G.: 1983, Chem. Phys. Lett. 102, p. 64.

188. Whyte, A.R. and Phillips, I.F.: 1983, Chem. Phys. Lett. 102, p. 451.

189. Meier, U., Grothier, H.H. and Just, Th.: 1984, Chem. Phys. Lett. 106, p. 97.

190. Wu, K.T.: 1984, Chem. Phys. Lett. 107, p. 405.

191. Kleinermanns, K. and Luntz, A.C.: 1983, J. Chem. Phys. 78, p. 3774.

192. Bermann, M.R. and Lin, M.: 1984, J. Chem. Phys. 81, p. 5743.

193. Persky, A. and Brioda, M.: 1984, J. Chem. Phys. 81, p. 4352.

194. Howard, M.J. and Smith, I.W.M.: 1982, J. Chem. Soc. Faraday Trans. 2, 78, p. 1403.

195. Howard, M.J. and Smith, I.W.M.: 1983, Prog. Reaction Kinetics 12, p. 55.

196. Miller, J.C. and Gordon, R.J.: 1983, J. Chem. Phys. 79, p. 1252.

197. Baulch, D.L., Co, R.A., Crutzen, P.J., Hampson, R.F., Kerr, J.A., Troe, J. and Watson, R.T.: 1982, J. Phys. Chem. Ref. Data 11, p. 327.

198. Cohen, N. and Westberg, R.R.: 1983, J. Phys. Chem. Ref. Data 12, p. 531.

199. Rau, S.N. and Truhlar, D.G.: 1983, J. Chem. Phys. 79, p. 6046.

200. Pamidimukkala, K.M. and Skinner, G.B.: 1982, J. Chem. Phys. 76, p. 311.

201. Lee, K.T., Bowman, J.M., Wagner, A.F. and Schatz, G.C.: 1982, J. Chem. Phys. 76, p. 3583 .

202. Clary, D.C.: 1984, Molec. Phys. 53, p. 3.

203. Bowman, J.M.: 1984, J. Chem. Phys. 81, p. 1739.

204. Clary, D.C. Werner, H-J.: 1984, Chem. Phys. Lett. 112, p. 346.

205. Bowman, J.M., Lee, K.T. and Walker, R.B.: 1983, J. Chem. Phys. 79, p. 3742.

206. Sun, J.C., Cho1, B.H., Poe, R.T. and Tang, K.T.: 1983, J. Chem. Phys. 78 p. 4523 and 79, p. 5376 . 
207. Borasin, J., Thomas, R. and Borassin, A.: 1983, J. Chem. Phys. 79, p. 1546. 208. Gerrity, D.P. and Valentine, J.J.: 1983, J. Chem. Phys. 79, p. 502.

209. Margolis, S., Garetz, B.A. and Sathyamurthy, N.: 1983, J. Chem. Phys. 79, p. 2736 .

210. Schwartz, S.D. and Miller, W.H.: 1983, J. Chem. Phys. 79, p. 3759.

211. Bowman, J.M. and Lee, K.T.: 1983, Chem. Phys. Lett. 94, p. 363.

212. Schatz, G.C.: 1983, Chem. Phys. Lett. 94, p. 183.

213. Abu Salbi, N., Kour1, D.J., Shuma, Y. and Baer, M.: 1984, Chem. Phys. Lett. 105 , p. 472.

214. Rozenshtein, V.B., Gershenzon, Yu. M., Ivanov, A.V., Kuchergavi1, S.I.: 1984, Chem. Phys. Lett. 105, p. 423.

215. Blais, N.C. and Truhlar, D.G.: 1983, Chem. k. Phys. Lett. 102, p. 120.

216. Choi, B.H., Poe, R.T. and Tang, K.T.: 1984, J. Chem. Phys. 81, p. 4979.

217. Pollak, E. and Wyatt, R.E.: 1984, J. Chem. Phys. 81, p. 1801.

218. Mayne, H.R.: 1984, J. Chem. Phys. 81, p. 2684.

219. Schatz, G.C., Hubbard, L.L., Dardi, P.S. and Mi11er, W.H.: 1984, J. Chem. Phys. 81, p. 231 .

220. Gerrity, D.P. and Valentine, J.J.: 1984, J. Chem. Phys. 81, p. 1298.

221. Connor, J.N.L. and Southa11, W.J.E.: 1984, Chem. Phys. Lett. 108, p. 527.

222. Shatz, G.C.: 1984, Chem. Phys. Lett. 108, p. 532.

223. Zamir, E., Levine, R.D. and Bernstein, R.D.: 1984, Chem. Phys. Lett. 107, p. 217 .

224. Glass, G.P. and Chatuverd1, B.K.: 1982, J. Chem. Phys. 77, p. 3478.

225. Flower, D.R. and Kirkpatrick, D.J.: 1982, J. Phys. B. 15, L11.

226. Baker, D.J. and Flower, D.R.: 1984, J. Phys. B. 17, p. 119.

227. Bel Bruno, J., Gelfand, J. and Rabitz, H.: 1983, J. Chem. Phys. 78, p. 3990.

228. Green, S. and Chapman, S.: 1983, Chem. Phys. Lett. 98, p. 467.

229. Storey, J.W., Watson, D.M. and Green, S.: 1982, Ap. J. 259, p. 647.

230. Bieniek, R.J. and Green, S.: 1983, Ap. J. 265, L29.

231. Elitzur, M.: 1983, Ap. J. 266, p. 609.

232. Green, S.: 1983, Ap. J. 277, p. 900.

233. Billing, G.d. and Cacciatore, M.: 1982, Chem. Phys. Lett. 86, p. 20.

234. Price, R.J., Clary, D.C. and Billing, G.D.: 1983, Chem. Phys. Lett. 101, p. 269 .

235. Teitelbaum, H.: 1984, Chem. Phys. Lett. 106, p. 69.

236. Bhattacharyaja, S.S. and Dickinson, A.S.: 1982, Astron. Ap. 107, p. 26.

237. Monterio, T.: 1984, MNRAS 210, 1.

238. Schinke, R., Meyer, H., Buck, U. and Diercksen, G.H.F.: 1984, J. Chem. Phys. 80 , p. 5518 .

239. Burns, M.J. and Coy, S.L.: 1984, J. Chem. Phys. 80, p. 3544.

240. Picard-Bersellini, A., Charneau, R. and Brechignac, P.L.: 1983, J. Chem. Phys. 79, p. 8900 .

241. Kohler, W.E. and Schaefer, J.: 1983, J. Chem. Phys. 78, p. 6602.

242. Beard, L.H. and Kouri, D.J.: 1983, J. Chem. Phys. 78, p. 220.

243. Jolicard, G. and Gros jean, A.: 1983, J. Chem. Phys. 78, p. 2327.

244. van Hemert, M.C.: 1983, J. Chem. Phys. 78, p. 2345.

245. Klassen, D.B.M., ter, Meulen, J.J. and Dymanus, A.: 1983, J. Chem. Phys. 78, p. 767 .

246. Wight, C.A., Magnotta, F. and Leone, S.R.: 1984, J. Chem. Phys. 81, p. 3951. 247. Billing, G.D. and Poulsen, L.L.: 1984, J. Chem. Phys. 81, p. 3866.

248. Roeging, A., Rabitz, H., Gelfand, J. and Miles, R.B.: 1984, J. Chem. Phys. 81, p. 820 .

249. Mehrotra, L.: 1984, Astrophys. Spa. Sci. 100, p. 129.

250. Drolshage, G. and Gianturco, F.A.: 1983, Molec. Phys. 48, p. 673.

251. Andres, J., Buck, U., Meyer, H. and Launay, J.M.: 1982, J. Chem. Phys. 76, p. 1417 .

252. Wood, C.F., Flynn, G.W. and Weston, R.E.: 1982, J. Chem. Phys. 77, p. 4776.

253. Andresen, P., Hausler, D. and Lulf, H.W.: 1984, J. Chem. Phys. 81, p. 571.

254. Andresen, P., Hausler, D., Lulf, H.W. and Kege1, W.H.: Astron. Ap. 138, L17.

255. Schinke, R. and Andresen, P.: 1984, J. Chem. Phys. 81, p. 5644. 
256. Devangan, D.P. and Flower, D.R.: 1982, MNRAS 199 p. 457.

257. Devangan, D.P. and Flower, D.R.: J. Phys. B. 17 p. 2157.

258. Flower, D.R. and Guilloteau, S.: 1982, Astron. Ap. 114 p. 238.

259. Sakimoto, K.: 1982, Chem. Phys. 68 p. 155; 1982, J. Phys. Soc. Japan 51, 2657; 1983, Chem. Phys. 79 p. 137; 1984, Chem. Phys. 85 p. 273.

260. Takayanagi, K.: 1982, J. Phys. Soc. Japan 51 p. 3337.

261. Su, T. and Chesnovich, W.J.: 1982, J. Chem. Phys. 76 p. 5183.

262. Bates, D.R.: 1982, Proc. Roy. Soc. A 384 p. 289.

263. Bates, D.R.: 1983, Chem. Phys. Lett. 97 p. 19.

264. Roberge, W.G. and Dalgarno, A.: 1982, Ap. J. 255 p. 1176.

265. Blais, N.C. and Truhlar, D.G.: 1982, Ap. J. 258, L79.

266. Blais, N.C. and Truhlar, D.G.: 1983, J. Chem. Phys. 78 p. 2388.

267. Lepp, S. and Shull, M.J.: 1983, Ap. J. 270 p. 578.

268. Federman, S.R. and Shipsey, F.J.: 1983, Ap. J. 269 p. 791.

\section{WORRING GROUP 4: STRUCTURE OF ATOMIC SPECTRA}

\section{A. Recent Laboratory Results and Regeareh in Progress}

A Bibliography on Atomic Energy Levels and Spectra covering the period July 1979 through December 1983 is scheduled for publication in late 1984(1). The references in Table 1 are thus limited to papers published or known to be in press in 1984; the selection was made from references giving data on energy levels, wavelengths, and line classifications for spectra of the elements $z \leq 30$. S. Johansson notes that the spectra under investigation in the Physics Department of Lund University, Sweden, include S III, IV, VII, VIII, IX; Cl VI, VII, VIII, $\mathrm{X}$; Sc V; the (Ne I) isoelectronic spectra Sc XII-Fe XVII; TI I; Cr I, II, V; Fe II, III; and several high-ionization spectra of $\mathrm{NI}$, the work on Fe III and the Ni spectra being done in collaboration with researchers in the Zeeman Laboratory, Amsterdam. Term analyses in progress at the Lund Institute of Technology, as reported by $W$. Persson, include Ne III and $K$ II, III. This group has recently completed analyses for $\mathrm{Kr} I V, \mathrm{Rb} V, \mathrm{Sr} V, \mathrm{VI}$ and has papers in preparation for $\mathrm{Rb}$ IV, Y VI, and Xe II. Fourier-transform spectrometric observations at the National Solar Observatory (KItt Peak) by J. Brault and collaborators are yielding more accurate and complete line lists for the first and second spectra of several elements, including $\mathrm{Mg}, \mathrm{Al}, \mathrm{Ti}, \mathrm{V}, \mathrm{Cr}, \mathrm{Fe}$, and $\mathrm{Ni}$. Ongoing extensions of the term analyses for some of these spectra were mentioned above; similar work is underway for Mg I, II, A1 I, II, and V I, II (E. Biémont, University of Liége) and for Fe I (R. C. M. Learner, Imperial College, London). B. C. Fawcett's current research at the Rutherford Appleton Laboratory, Oxfordshire, England, includes work on the oxygen-1ike spectra P VIII through Fe XIX.

\section{B. Compilations, Isoelectronic-Sequence Observations and Predictions}

The NBS Atomic Energy Levels Data Center published energy-levels compllations for all the spectra of $\mathrm{Fe}(29)$ and $\mathrm{SI}(30)$. Revised and updated energy-levels compllations for the 235 spectra of the ten iron-group elements $K$ through $\mathrm{Ni}$ will appear in a single volume in $1985(31)$. A compilation of levels for the $P$ spectra has also been submitted(32), and C. E. Moore has prepared new tables of levels and multiplets for 0 IV and 0 III(33).

R. L. Kelly's revised compilation of atomic lines below 2000 A is scheduled for publication in 1985(34). These tables supersede a similar 1973 compilation and cover the same elements, $\mathrm{H}$ through $\mathrm{Kr}$. Fawcett's review(28) includes tables of observed forbidden lines for ions stripped to the $n=2$ and $n=3$ shells. Some of the tables of atomic wavelengths in the CRC Handbook were revised and updated for the latest edition(35); about 45000 lines from the first five spectra of all elements in all wavelength ranges are included. 